\title{
ON THE QUANTIFICATION OF DISCRETIZATION UNCERTAINTY: COMPARISON OF TWO PARADIGMS
}

\section{JULIEN BECT ${ }^{1}$, SOULEYMANE ZIO ${ }^{1}$, GUILLAUME PERRIN ${ }^{2}$, CLAIRE CANNAMELA $^{2}$ AND EMMANUEL VAZQUEZ ${ }^{1}$}

${ }^{1}$ Université Paris-Saclay, CNRS, CentraleSupélec, Laboratoire des Signaux et Systèmes (L2S), 91190, Gif-sur-Yvette, France.

${ }^{2}$ Commissariat à l'énergie atomique et aux énergies alternatives (CEA), CEA/DAM/DIF, 91297, Arpajon, France.

* Corresponding author. E-mail: julien.bect@centralesupelec.fr

Key words: Quantification of uncertainty, Discretization, Grid convergence index, Gaussian processes

\begin{abstract}
The use of simulation has spread to all areas of engineering and science, and the use of numerical models based on partial differential equations has thus multiplied. The resolution of these models is generally based on the discretization of the space in which the solutions to the equations under consideration are sought. The finite differences method or the finite elements method are two examples of such a discretization. This discretization simplifies the solving but implies a form of uncertainty on the value of any quantity of interest. To quantify this discretization uncertainty, the grid convergence index (GCI), based on the Richardson extrapolation technique, is now standard in the Verification and Validation (V\&V) literature. But alternative approaches were also proposed in the statistical literature, such as Bayesian approaches with Gaussian process models. The objective of this work is to compare on a standard test case from the literature (Timoshenko's beam) the well-established GCI-based approach to the - younger-Bayesian approach for the quantification of discretization uncertainty.
\end{abstract}

\section{INTRODUCTION}

Numerical models based on partial differential equations (PDE), or integro-differential equations, are ubiquitous in engineering and science, making it possible to understand or design systems for which physical experiments would be expensive - sometimes impossible - to carry out. Such models usually construct an approximate solution of the underlying continuous equations, using discretization methods such as finite differences or the finite elements method. The resulting discretization error introduces a form of uncertainty on the exact but unknown value of any quantity of interest (QoI), which affects the predictions of the numerical model alongside other sources of uncertainty such as parametric uncertainty or model inadequacy (see, e.g., the typology proposed by [6]). The present article deals with the quantification of this discretization uncertainty, which is an instance of the more general concept of numerical uncertainty (see, e.g., 

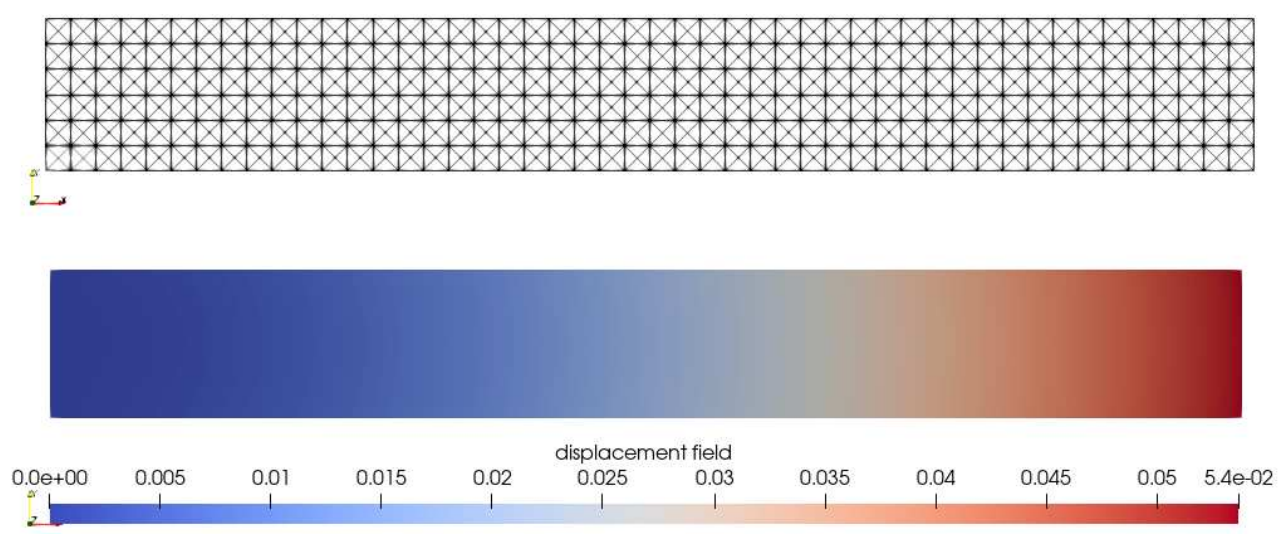

Figure 1: Timoshenko's beam. The isotropic rectangular beam is fixed at its left end, and a vertical pressure field is applied at the other end (see Section 4.1 for details). Top: discretization mesh with $L=48 \mathrm{~m}, D=6 \mathrm{~m}$ and $h=1 \mathrm{~m}$. Bottom: displacement field for $\nu=0.1$ and $P=800$.

chapters 7 and 8 of $[7]$ and references therein).

As an example, consider the "Timoshenko beam" problem (see Figure 1), and assume that the QoI is the vertical displacement of the beam, measured at a given location. The equations of linear elasticity describing this problem are discretized in space using triangular mesh, the

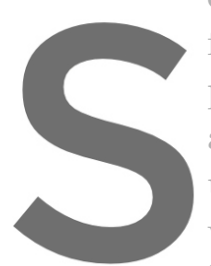
finesse of which is controll problem (beam dimensi approximate value $f(h)$ used for this run. For convergent discretization value of the QoI for the exact sth it is usually possible, however, to run the PI
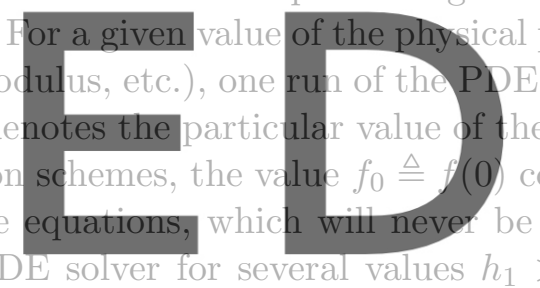

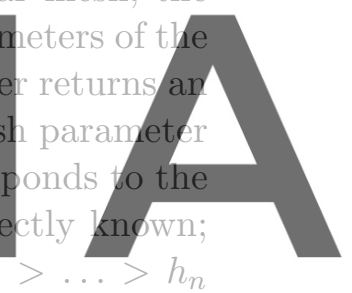

$h_{n}$

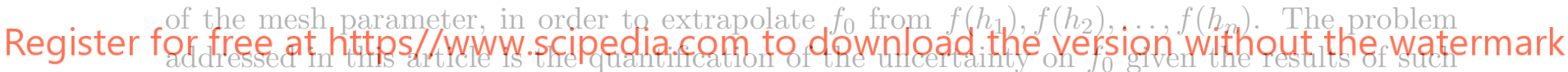
a grid refinement study.

A first approach to this problem, now standard in the V\&V (Verification and Validation) literature [7], uses the grid convergence index (GCI) originally proposed by Roache [11] in the field of computational fluid dynamics (CFD). It is based on a simple but clever reinterpretation of the Richardson extrapolation technique, which has a long history in numerical analysis, going back to the original work of Richardson $[9,10]$. The key underlying assumption is that the discretization error behaves as

$$
f(h)-f_{0}=A h^{p}+o\left(h^{p}\right),
$$

where $A$ and $p$ are two parameters that are usually considered unknown (although a theoretical value of $p$, known as the "formal order" of convergence, is available in some situations). Roache proposed a method that uses (1), not to produce an extrapolated value $\widehat{f}_{0}$ as is usually done in numerical analysis, but to construct an "error band [...] in which the reader/user can have some practical level of confidence" [11, p. 407]. The word "confidence", however, must be taken 
here - and later in this article when referring to the GCI approach - in its casual, non-statistical acceptation, and not as an indication of a "well-founded probability statement" [11, p. 407 again].

Another approach was more recently introduced in the statistical literature [19], in the context of a general trend of research on the design and analysis of computer experiments using Gaussian process (GP) models, initiated at the end of the 80's by Sacks and co-authors [5, 14, 15, 21], and first applied to the analysis of computer experiments with multiple levels of fidelity - also known as "multi-fidelity" computer experiments - by Kennedy and O'Hagan [6]. This approach relies on the Bayesian machinery: prior knowledge on the function $f: h \mapsto f(h)$ is encoded by a probability distribution - a GP model for the sake of tractability - , which gives birth, when combined with simulation results, to a posterior distribution that quantifies the resulting uncertainty on $f$. In particular, this posterior distribution can be used to make "well-founded probability statements" (in a Bayesian sense) on the unknown QoI $f_{0}$.

The objective of this article is to present and compare these two paradigms for the quantification of discretization uncertainty, which have been developed in different scientific communities, and to assess the potential of the Bayesian approach to provide a replacement for the well-established GCI-based approach, with better probabilistic foundations. The article is organized as follows. Section 2 provides a short introduction to both paradigms. Section 3 provides theoretical results about several classes of covariance functions that can be considered for the Bayesian approach. Section 4 presents the result of our numerical results on a standard test case from the literature

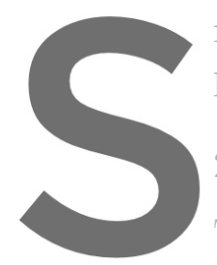
namely, Timoshenko's beam. Finally, Section
possible directions for future work.
2 DISCRETIZATION UNCERTAINTY This section summarizes the two main paradig
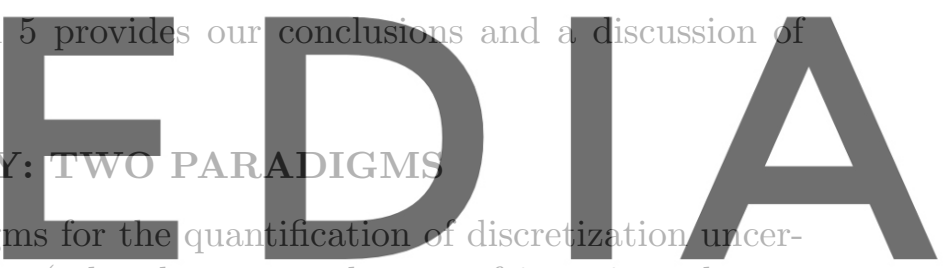

tainty. Other sources of numerical uncertainty (related, e.g., to the use of iterative schemes

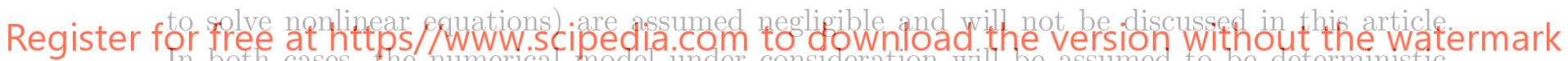
In both cases, the numerical nodel under consideration will be assumed to be deterministic. (The second approach can also deal with stochastic simulators; see, e.g., the work of Stroh and co-authors $[17,18]$.

\subsection{Numerical analysis approach}

In the scientific computing literature, the most commonly used method for the quantification of discretization uncertainty is the GCI (Grid Convergence Index) method, proposed by Roache [11-13] and reviewed, e.g., in Chapter 8 of Roy and Oberkampf's book [7]. It is based on a re-interpretation of Richardson's extrapolation procedure $[9,10]$, a well-established idea in numerical analysis. More precisely, assume that the QoI satisfies Equation (1) for some $A \in \mathbb{R}$ and $p>0$. The values $f_{0}, A$ and $p$ which appear in Equation (1) can be estimated by evaluating $f$ at the different mesh sizes $h_{1}<h_{2}<h_{3}$ (often called fine, medium and coarse) and then solving the system of nonlinear equations obtained by neglecting higher order terms:

$$
f\left(h_{k}\right)=f_{0}+A h_{k}^{p}, \quad 1 \leq k \leq 3 .
$$


The solution can be written explicitly if the mesh sizes satisfy $h_{2} / h_{1}=h_{3} / h_{2}=r$ for some $r>1$ :

$$
\begin{aligned}
\widehat{p} & =\ln \left(\frac{f\left(h_{3}\right)-f\left(h_{2}\right)}{f\left(h_{2}\right)-f\left(h_{1}\right)}\right) / \ln (r), \\
\widehat{A} & =\frac{f\left(h_{2}\right)-f\left(h_{1}\right)}{h_{1}^{\widehat{p}}\left(r^{\widehat{p}}-1\right)} \\
\widehat{f_{0}} & =f\left(h_{1}\right)+\frac{f\left(h_{1}\right)-f\left(h_{2}\right)}{r^{\widehat{p}}-1},
\end{aligned}
$$

yielding the approximation

$$
f(h) \approx \widehat{f}(h)=\widehat{f_{0}}+\widehat{A} h^{\widehat{p}} .
$$

Until now what we have described is nothing more than Richardson's extrapolation method. The GCI method takes the idea one step further, by considering a confidence interval centered around the value of highest fidelity (a.k.a. fine grid solution):

$$
C I_{\mathrm{GCI}}=\left[f\left(h_{1}\right)-U ; f\left(h_{1}\right)+U\right]
$$

for some $U>0$. Assuming that Equation (3) actually provides an exact representation of $f$-in other words, that there are no higher-order terms-- it is then easy to see that the exact solution belongs to the interval if, and only if,

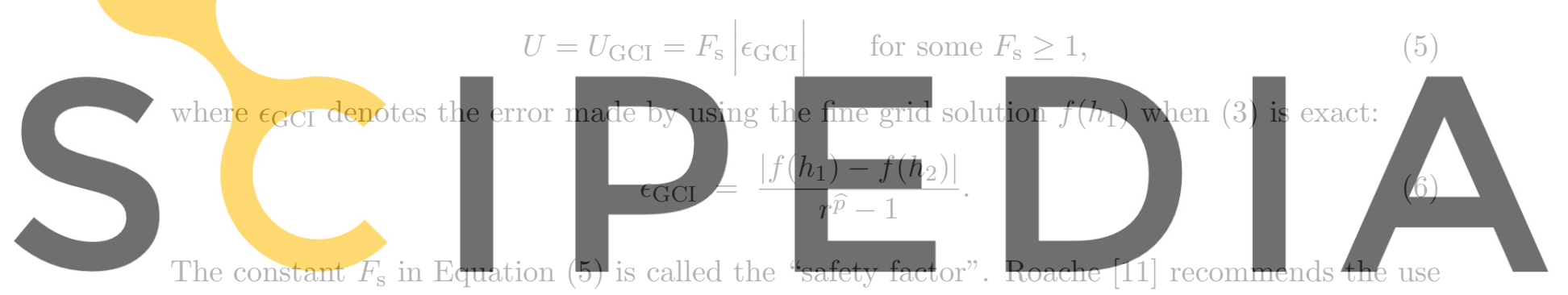

of $F_{\mathrm{S}}=3$ in general, except when the value of $p$ is known beforehand from numerical analysis

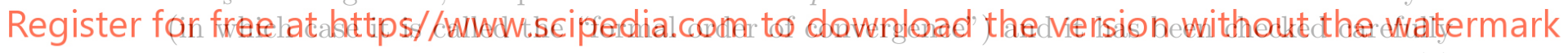

that the solutions have been computed in the "asymptotic range" where the approximation (3)

(with $\widehat{p}=p$ known) is accurate; in which case the value $F_{\mathrm{s}}=1.25$ is recommended.

Figure 2 illustrates the GCI method with an example taken from the Timoshenko beam problem (see Section 4.1 for a full description). Observe in particular the interval is indeed centered around the fine grid solution (and not around the extrapolated solution): it is important to keep in mind that the GCI approach uses Richardson's extrapolation technique to define a confidence interval, but not to actually extrapolate to a more accurate solution.

\subsection{Probabilistic (Bayesian) approach}

In 2014, Tuo and co-authors [19] introduced an approach to deal with ${ }^{1}$ discretization uncertainty using a concept of Bayesian multifidelity $[6,8]$. Under this approach, the QoI $f_{0}$ is assumed to be

\footnotetext{
${ }^{1}$ To be precise, [19] considers a QoI that depends on a vector $x$ ranging in a set $\mathbb{X}$ corresponding to the input space of a numerical simulator. For a given $x \in \mathbb{X}$, the objective is to infer $f(x, 0)$ given simulation results $f\left(x_{1}, h_{1}\right), \ldots, f\left(x_{n}, h_{n}\right)$. Here, we focus on a more specific problem, that is, grid refinement studies, where $x$ is fixed and $h$ varies.
} 

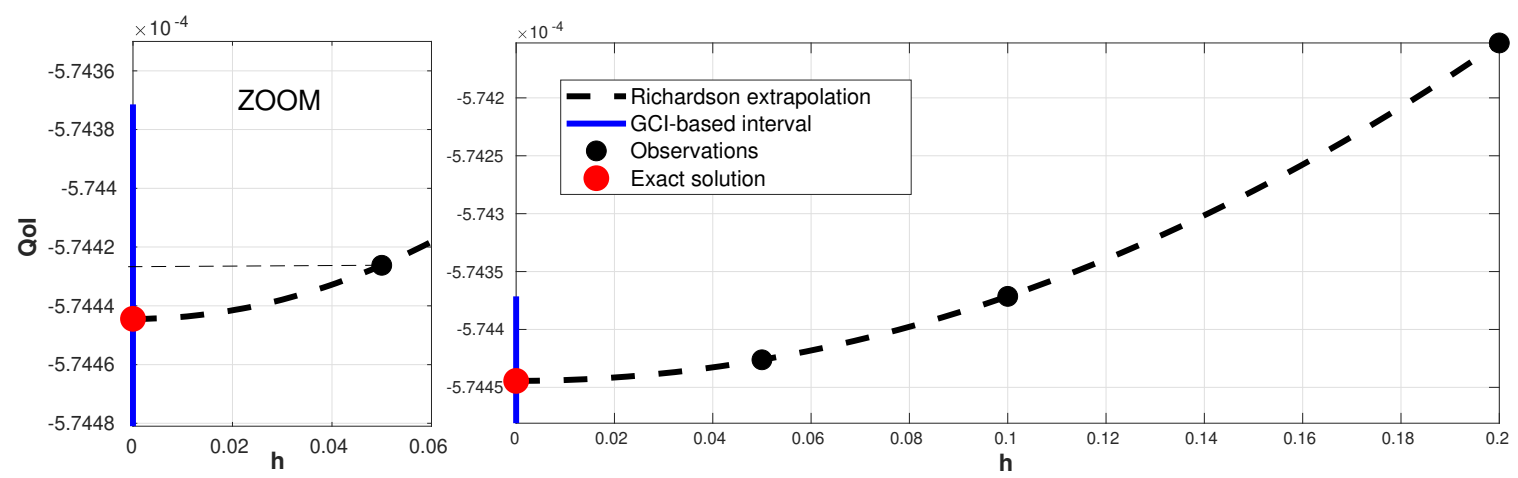

Figure 2: Illustration of the GCI method on the Timoshenko beam problem, with $F_{\mathrm{s}}=3$. The QoI is the vertical displacement at $x=10 \mathrm{~m}$. Three grid sizes are used: $h_{1}=1 / 20, h_{2}=1 / 10$, and $h_{3}=1 / 5$.

a sample value of a random variable $\xi_{0}$, which models uncertainty about the unknown value $f_{0}$. The prior distribution of this random variable - i.e., its distribution before any actual evaluation of the numerical model is made - encodes prior beliefs about the plausible values of $f_{0}$.

In the domain of design and analysis of computer experiments, it is customary to use Gaussian prior distributions for the outputs of (deterministic) numerical simulators, since Gaussian distributions are conjugate priors, which conveniently lead to Gaussian posterior distributions.

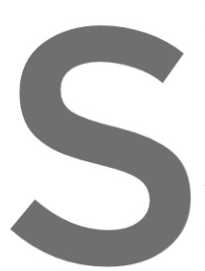

Following [19], we mode
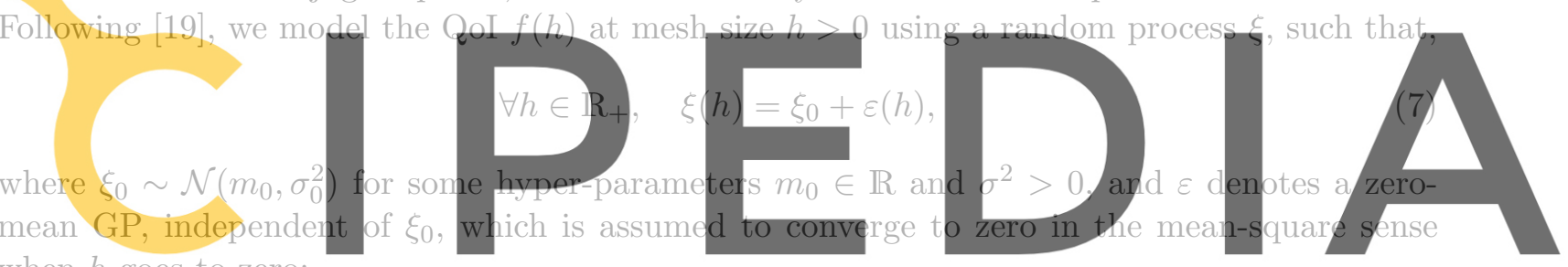

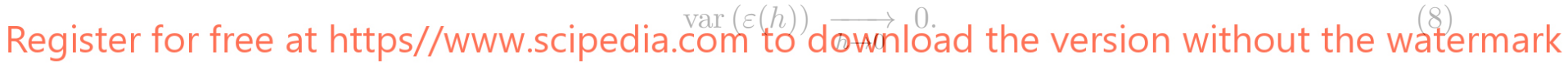

The GP $\varepsilon$ corresponds to the error of discretization - in other words, $\varepsilon$ models the loss of

fidelity as $h$ increases. The distribution of $\varepsilon$ will be denoted by $\operatorname{GP}\left(0, k_{\varepsilon}\right)$, where $k_{\varepsilon}$ stands for the covariance function of $\varepsilon$, which is such that $\lim _{h, h^{\prime} \rightarrow 0} k_{\varepsilon}\left(h, h^{\prime}\right)=0$, due to (8). Note that, conditional on $\xi_{0}, \xi$ is a non-stationary GP with mean function $\xi_{0} \mathbb{1}_{\mathbb{R}_{+}}$and covariance function $k_{\varepsilon}$.

In practice, it is convenient to assume an improper uniform distribution $\mathcal{U}_{\mathbb{R}}$ for $\xi_{0}$, which may be thought as taking the limit $\sigma_{0}^{2} \rightarrow \infty$ (for any fixed $m_{0} \in \mathbb{R}$ ). In this case, the posterior distribution of $\xi$ is given by the equations of ordinary kriging, which are recalled in the following.

Proposition 1 (Ordinary kriging). Let $\mathbb{X}$ denote a set and $k$ a covariance function on $\mathbb{X}$. Let $\xi$ denote an (improper) $G P$ on $\mathbb{X}$, such that $\xi \mid m \sim \operatorname{GP}(m, k)$ and $m \sim \mathcal{U}_{\mathbb{R}}$. Let $n \geq 1$ and $h_{1}, \ldots, h_{n} \in \mathbb{X}$. Then, for all $h \in \mathbb{X}$,

$$
\xi(h) \mid \xi\left(h_{1}\right), \ldots, \xi\left(h_{n}\right) \sim \mathcal{N}\left(\widehat{\xi}_{n}(h), s_{n}^{2}(h)\right),
$$

with 


$$
\begin{aligned}
& \widehat{\xi}_{n}(h)=\widehat{m}_{n}+k_{n}(h)^{\top} K_{n}^{-1}\left(\underline{\xi}_{n}-\widehat{m}_{n} \mathbb{1}_{n}\right), \\
& s_{n}^{2}(h)=k(h, h)-k_{n}(h)^{\top} K_{n}^{-1} k_{n}(h)+\frac{\left(1-k_{n}(h)^{\top} K_{n}^{-1} \mathbb{1}_{n}\right)^{2}}{\mathbb{1}_{n}^{\top} K_{n}^{-1} \mathbb{1}_{n}},
\end{aligned}
$$

where $\underline{\xi}_{n}=\left(\xi\left(h_{1}\right), \ldots, \xi\left(h_{n}\right)\right)^{\top}$ is the vector of observations, $k_{n}(h)$ the correlation vector between $\xi(h)$ and $\underline{\xi}_{n}, K_{n}$ the covariance matrix of $\underline{\xi}_{n}, \mathbb{1}_{n}=(1, \ldots, 1)^{\top}$, and $\widehat{m}_{n}=\mathbb{1}_{n}^{\top} K_{n}^{-1} \underline{\xi}_{n} / \mathbb{1}_{n}^{\top} K_{n}^{-1} \mathbb{1}_{n}$ the weighted least squares estimate of $m$.

Proposition 1 provides us with a method to build confidence interval about $f_{0}$ from simulations at mesh sizes $h_{1}, \ldots, h_{n}$. The procedure to compute confidence (the term credibility would be preferred under a Bayesian terminology) intervals consists of the following steps:

1. Given simulation results $f\left(h_{1}\right), \ldots, f\left(h_{n}\right)$, and the choice of a parameterized covariance function $k$, which will be discussed in Section 3, estimate the parameters of $k$ using a maximum likelihood approach (see Section 4$)^{2}$

2. Using the covariance function $k$ estimated at the previous step and the corresponding GP model $\xi$ for $f$, compute the Gaussian posterior distribution of the QoI $\xi_{0}=\xi(0)$. This gives a posterior mean value $\widehat{\xi}_{n}(0)$, which corresponds to $\widehat{m}_{n}$ since $k$ goes to zero at the origin, and a posterior variance $s_{n}^{2}(0)$. (Of course, the user can in fact obtain the posterior mean and the posterior variance at any $h$.)
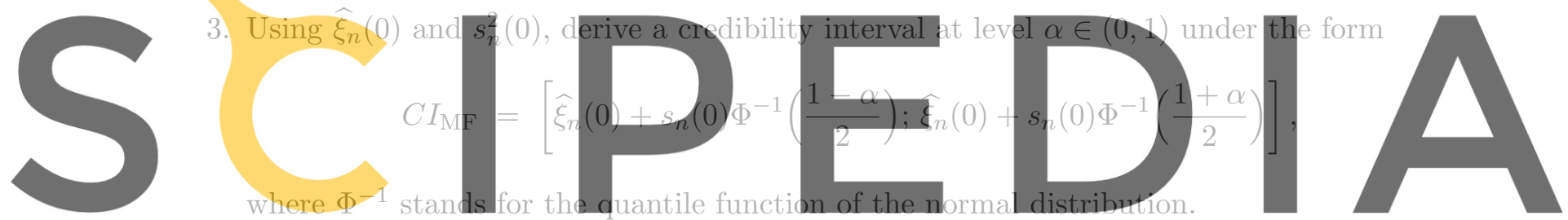

The procedure is illustrated on Figure 3. Observe that, in contrast with the GCI approach,

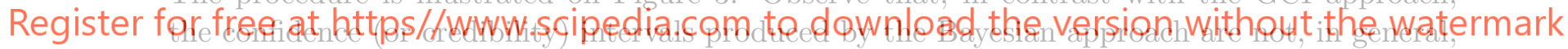

centered around the highest-fidelity value. They are centered instead around the extrapolated value, that is, the posterior mean at $h=0$. In the special case of the Brownian-like covariance function of [19] (see below), however, the extrapolated value coincides with the observation of highest fidelity.

\section{COVARIANCE FUNCTIONS}

In this section, we tackle from a theoretical point of view the question of choosing a suitable covariance function for the GP model in the Bayesian approach of Section 2.2, when prior evidence indicates that the unknown function $f$ obeys (1) as in the GCI approach. More precisely, assuming that $\varepsilon$ is a zero-mean GP with continuous sample paths ${ }^{3}$ on $[0,+\infty)$, we will provide

\footnotetext{
${ }^{2}$ The proposed procedure does not take into account the uncertainty resulting from the estimation of the parameters of the covariance. This could be investigated in future work.

${ }^{3}$ Let us recall a classical sufficient condition for the sample path continuity of a zero-mean GP on $\mathbb{X} \subset \mathbb{R}^{d}$ [see, e.g., 1, Theorem 3.4.1]: if there exist $C>0$ and $\eta>0$ such that $k(x, x)+k(y, y)-2 k(x, y) \leq \frac{C}{|\log \|x-y\||^{1+\eta}}$ for all $x, y \in \mathbb{X}$, then there exists a version of $\xi$ with continuous sample paths.
} 

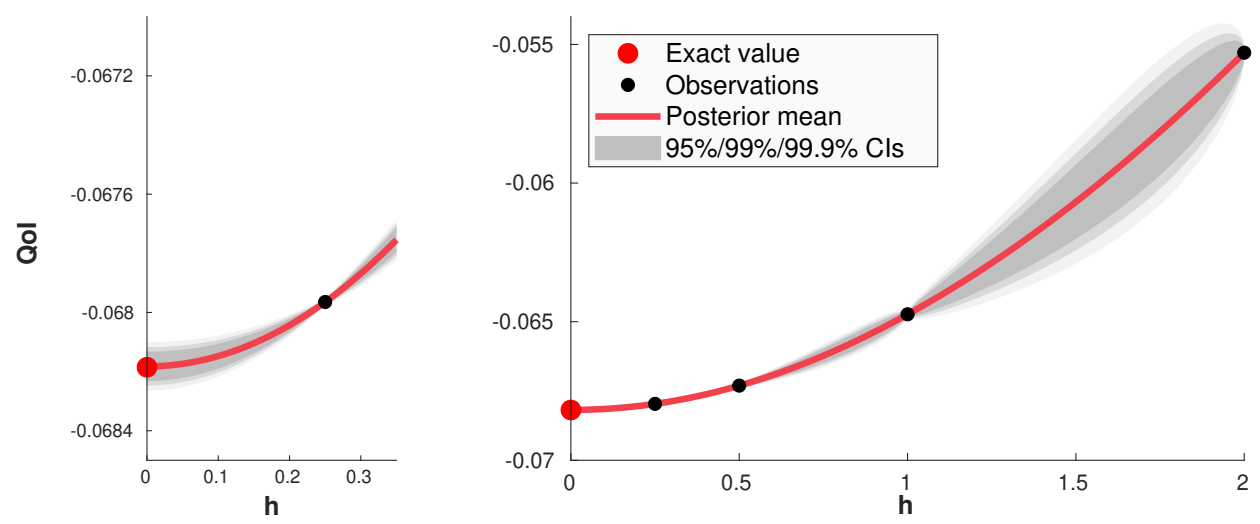

Figure 3: Illustration of the Bayesian approach. Left: global view. Right: zoom at $h=0$. Four observations, at $h=2,1,0.5,0.25$. GP model: TWY2 covariance function (see Section 3) with $\sigma=510^{-3}$, $L=4$ and a Matérn-1/2 (exponential) stationary covariance function with range parameter $\rho=200$.

conditions on the covariance function $k$ under which the property

$$
(\mathrm{R}): \quad \exists A \neq 0, \exists p>0, \quad \varepsilon(h)=A h^{p}+o\left(h^{p}\right),
$$

holds almost surely (where $A$ and $p$ are allowed to depend on the particular sample path that

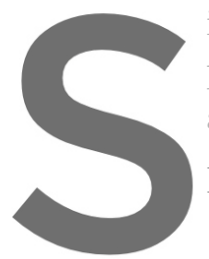
is considered). Proofs

publication. Our first

and shows that this mor

Proposition 2. Assum
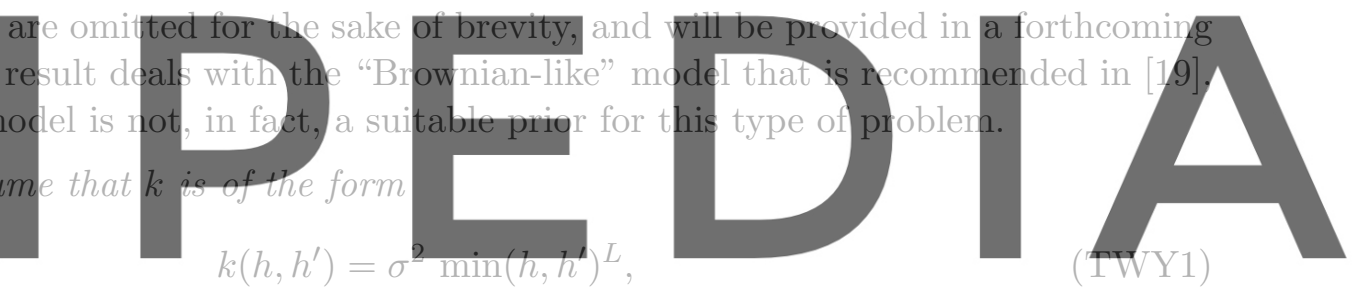

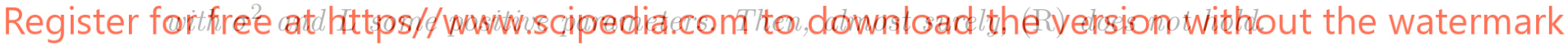

Our second result deals with the second model that is considered - but not advocated - in [19].

Proposition 3. Assume that $k$ is of the form

$$
k\left(h, h^{\prime}\right)=\sigma^{2}\left(h h^{\prime}\right)^{L / 2} c\left(h-h^{\prime}\right),
$$

where $\sigma^{2}$ and $L$ are positive parameters, and $c$ is the stationary correlation function of a GP with continuous sample paths. Then $(\mathrm{R})$ holds almost surely with $p=\frac{L}{2}$.

This model is thus suitable for the problem under consideration, for any value of $p$. Note that, for a given value of $p$, significant modeling flexibility remains, through the choice of the stationary correlation $c$. For instance, the squared exponential (a.k.a. Gaussian) correlation can be used as in [19], but rougher correlation functions, such as the Matérn family of correlation functions [16], can be considered as well.

Our last result deals with covariance functions of the form

$$
k\left(h, h^{\prime}\right)=\sigma^{2}\left[1+c\left(h-h^{\prime}\right)-c(h)-c\left(h^{\prime}\right)\right],
$$


where $c$ is a stationary correlation function. This is the covariance function of $\tilde{\xi}-\tilde{\xi}(0)$, where $\tilde{\xi}$ is a stationary GP with covariance function $k_{\text {stat }}\left(h, h^{\prime}\right)=\sigma^{2} c\left(h-h^{\prime}\right)$.

Proposition 4. Assume that $k$ is of the form (11), where $\sigma^{2}$ is a positive parameter and $c$ is the stationary correlation function of a GP with continuous sample paths.

i) If property $(\mathrm{R})$ holds almost surely, then $p \leq 1$ almost surely.

ii) If $c$ is the covariance function of a GP with differentiable sample paths, then there exists a version of $\varepsilon$ such that property $(\mathrm{R})$ holds almost surely with $p=1$.

Such covariance functions are thus, in principle, only suitable for $p \leq 1$. (We even conjecture that $4 . \mathrm{i}$ actually holds with " $p=1$ " instead of " $p \leq 1$ ").

\section{NUMERICAL EXPERIMENTS}

\subsection{Test case}

We consider the quasi-static deformation of an isotropic rectangular beam. $\Omega=[0, L] \times$ $[-D / 2, D / 2]$ is the domain characterizing the initial position of the beam, $\Gamma_{\text {left }}$ and $\Gamma_{\text {right }}$ are the left and right sides of the beam, and $\Gamma_{\text {other }}$ is the union of the two other sides of the beam. The beam is supposed to be fixed at one end, and a specific vertical pressure field, denoted by $p$, is applied at the other end. Under linear elasticity, and neglecting the gravity forces and the atmospheric pressure, it can be shown [20] that the displacement field in each point of the beam,

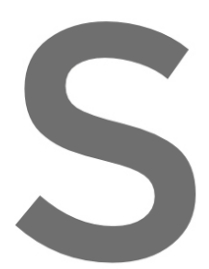
written $\mathbf{u}=\left(u_{1}, u_{2}\right)$.
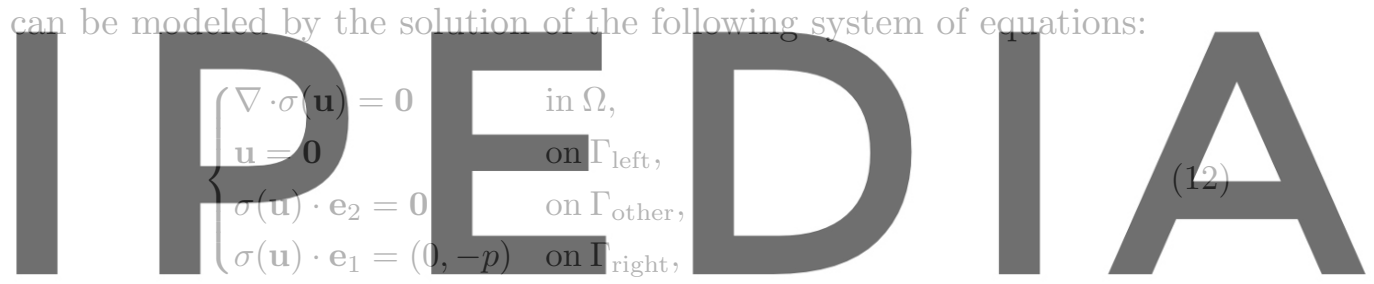

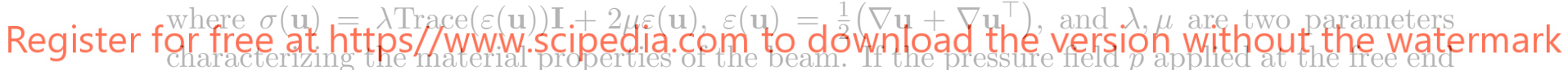
of the beam $\left(x_{1}=L\right)$ is given by $p=\frac{P}{2 I}\left(\frac{D^{2}}{4}-x_{2}^{2}\right)$ with $I=\frac{1}{12} D^{3}$ the moment of inertia of the beam, there exists an explicit solution for $\mathbf{u}$ :

$$
\begin{aligned}
& u_{1}\left(x_{1}, x_{2}\right)=\frac{P x_{2}}{6 E^{*} I}\left[\left(6 L-3 x_{1}\right) x_{1}+\left(2+\nu^{*}\right) x_{2}^{2}-\frac{3 D^{2}}{2}\left(1+\nu^{*}\right)\right], \\
& u_{2}\left(x_{1}, x_{2}\right)=\frac{P}{6 E^{*} I}\left[3 \nu^{*} x_{2}^{2}\left(L-x_{1}\right)+\left(4+5 \nu^{*}\right) \frac{D^{2} x_{1}}{4}+\left(3 L-x_{1}\right) x_{1}^{2}\right],
\end{aligned}
$$

with $\lambda=\frac{E \nu}{(1+\nu)(1-2 \nu)}, \mu=\frac{E}{2(1+\nu)}, E^{*}=\frac{E}{1-\nu}$ and $\nu^{*}=\frac{\nu}{1-\nu}$. In the numerical experiments, the values of $E, L$ and $P$ will be fixed to $3 \times 10^{7} \mathrm{~N} / \mathrm{m}^{2}, 48 \mathrm{~m}$ and $1000 \mathrm{~N}$ respectively, whereas the values of $D$ and $\nu$ will vary. Finally, we will consider as QoIs the vertical displacement at four different positions:

$$
f_{j}=u_{2}\left(x_{p_{j}}, 0\right), \quad 1 \leq j \leq 4,
$$

with $x_{p_{1}}=10 \mathrm{~m}, x_{p_{2}}=20 \mathrm{~m}, x_{p_{3}}=30 \mathrm{~m}$ and $x_{p_{4}}=48 \mathrm{~m}$. 


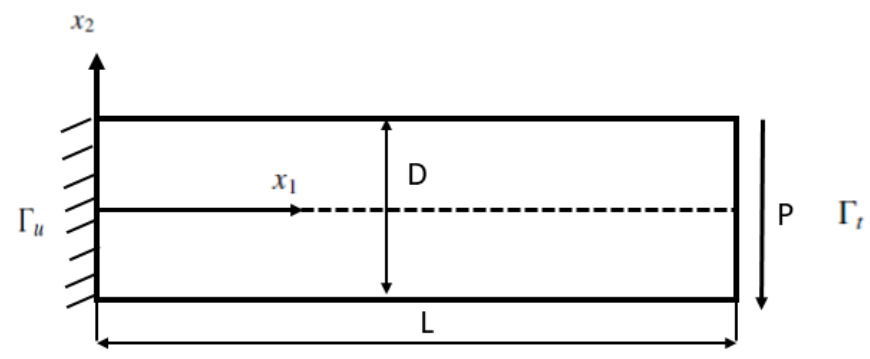

Figure 4: Timoshenko Beam

Remark 1. Augarde and Deeks [3] discuss alternative formulations of the beam problem with more realistic boundary conditions (but no analytical solutions).

\subsection{Experimental setup}

We consider six possible values for the height: $D \in\{2 \mathrm{~m}, 4 \mathrm{~m}, \ldots, 12 \mathrm{~m}\}$ and nine values for the Poisson ratio: $\nu \in\{0,0.10,0.15,0.20,0.25,0.30,0.35,0.40,0.45\}$, thereby creating 54 instances of the Timoshenko beam problem. The PDE is solved in FEniCS [2], using a finite element method with a regular triangular mesh (see Figure 1).

Two methods for constructing confidence intervals about the exact value of QoIs are compared:

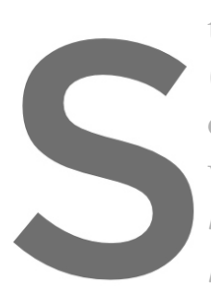
the GCI approach (see Section 2.2) with $\alpha$ chosen in order to const value in most (if not $h_{j}^{\mathrm{GCI}}=2^{j-3} \cdot h_{3}^{\mathrm{GCI}}, 1$
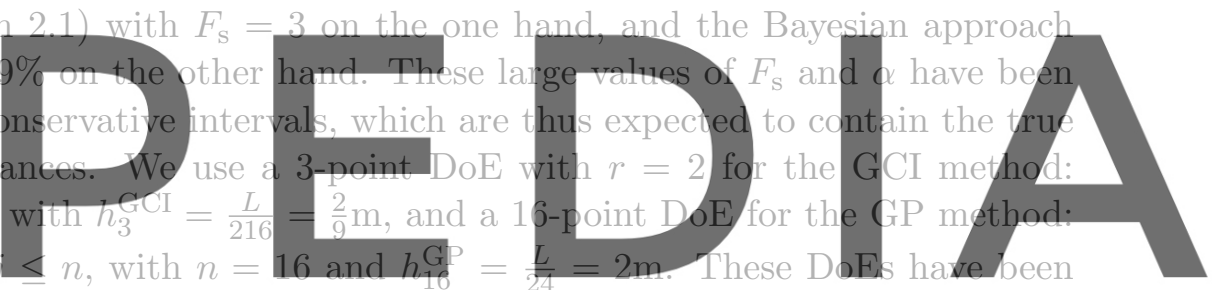

chosen in order to have similar computation times for both methods.

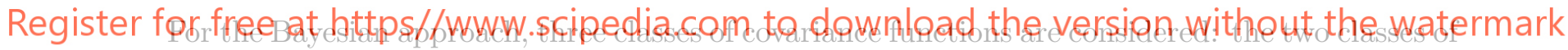
covariance functions proposed by [19] and described in Propositions 2-3, denoted as TWY1 and TWY2 respectively (for "Tuo, Wu and Yu"), and the one described in Proposition 4, denoted by STZ (for "STationary minus the value at Zero"). For the TWY1 and TWY2 classes, the parameter $L$ is either set to $L=1$ or $L=2$, or estimated along with the others by restricted maximum likelihood. For the TWY2 and STZ classes, a stationary correlation function must be specified: we consider as possible choices the Matérn covariance function with regularity $\nu=\frac{1}{2}$ (a.k.a. exponential covariance function), $\nu=\frac{3}{2}$ or $\nu=\frac{5}{2}$, the Matérn covariance function with estimated regularity, and the Gaussian (a.k.a. squared exponential) covariance function. All in all, this gives us a total of 23 covariance models to be compared ( 3 for the TWY1 class, 15 for the TWY2 class and 5 for the STZ class). GP modeling computations are carried out using the STK toolbox [4].

\subsection{Results}

Figure 5 presents the average performance of all the $23+1=24$ methods on the 54 instances of the problem. Two performance metrics are considered: the coverage of the interval, which is 

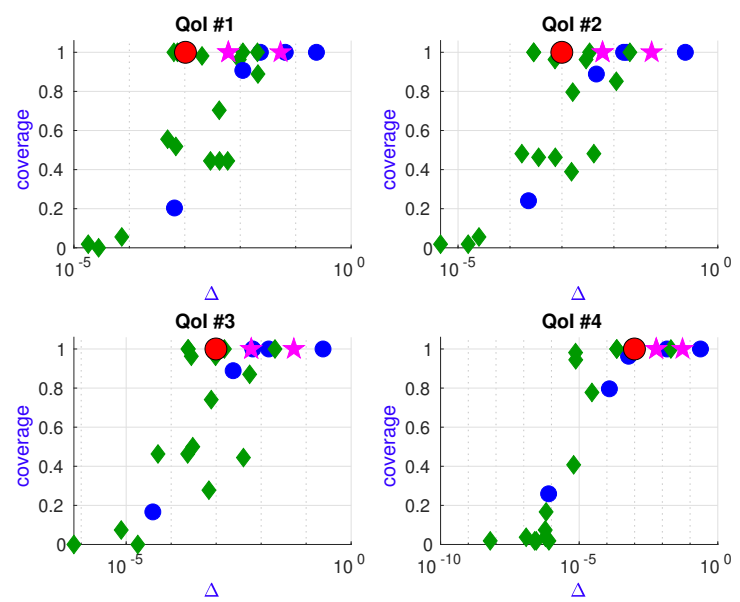

Figure 5: Empirical coverage and average interval half-width for the $23+1=24$ methods. Red disks: GCI-based intervals. Pink stars: TWY1. Green diamonds: TWY2. Blue disks: STZ.

the proportion of instance where the interval contains the true value, and the average interval half-width (IWH), denoted by $\Delta$. As expected, the GCI interval is conservative its empirical coverage is actually equal to $100 \%$ in this experiment, for all four QoIs. The results are much more contrasted for the Bayesian approach, however: depending on the covariance function, the

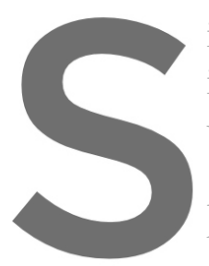
interval is either overdont interval), or - and this with a good coverage.

A closer look at the results allows to identify some promising the Bayesian approach.
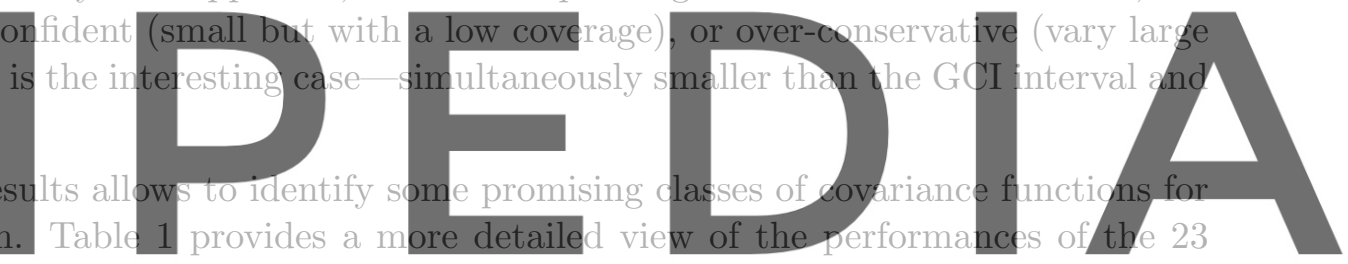

covariance models, focusing on the most promising ones - defined as those which obtained both

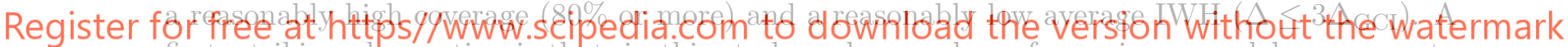

first, striking observation is that, in this study, only one class of covariance models manages to

deliver intervals that are simultaneously smaller than the GCI interval and with a good coverage:

the TWY2 class, and more precisely the TWY2 class with a weakly-regular stationary correlation function (Matérn with $\nu=\frac{1}{2}$ or $\nu=\frac{3}{2}$ ).

Concerning the decay parameter $L$, the best performances are obtained when it is fixed a priori to $L=4$, or estimated. This is consistent with Proposition 3, since it corresponds to a quadratic convergence $\left(p=\frac{L}{2}=2\right)$, which is the actual convergence rate in this problem. Note that, with the Matérn-3/2 covariance function, the results when $L$ is estimated are not as good as when it is fixed to the true value, which is not surprising per se, but suggests that there might be room for improvement in the parameter selection procedure. The fact that covariance functions from the TWY1 and STZ classes do not lead to satisfactory intervals is also consistent with the theoretical results of Section 3. Indeed, from a sample path point of view, neither of these classes provides a suitable prior for a quadratic convergence at $h=0$ (see Propositions 2 and 4 , respectively). 


\begin{tabular}{|c|c|c|c|c|}
\hline covariance & QoI \#1 & QoI \#2 & QoI \#3 & QoI \#4 \\
\hline TWY2 $\left(\mathcal{M}_{1 / 2}, L=\widehat{L}\right)$ & $1.000,0.716$ & $1.000,0.287$ & $1.000,0.239$ & $1.000,0.228$ \\
\hline TWY2 $\left(\mathcal{M}_{1 / 2}, L=4\right)$ & $1.000,0.619$ & $1.000,0.289$ & $1.000,0.243$ & $1.000,0.232$ \\
\hline TWY2 $\left(\mathcal{M}_{3 / 2}, L=\widehat{L}\right)$ & : & $0.963,2.931$ & $0.963,0.980$ & $0.944,0.008$ \\
\hline TWY2 $\left(\mathcal{M}_{3 / 2}, L=4\right)$ & $0.981,1.989$ & $0.963,0.739$ & $0.963,0.284$ & $0.981,0.008$ \\
\hline TWY2 $\left(\mathcal{M}_{3 / 2}, L=2\right)$ & : & $\theta$ & $1.000,1.543$ & $1.000,0.637$ \\
\hline $\operatorname{STZ}\left(\mathcal{M}_{5 / 2}\right)$ & 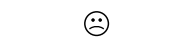 & : & (2) & $0.963,0.614$ \\
\hline $\operatorname{STZ}\left(\mathcal{M}_{\widehat{\nu}}\right)$ & $\odot$ & 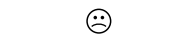 & $0.889,2.414$ & 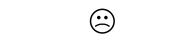 \\
\hline All the other cases & : & : & : & : \\
\hline
\end{tabular}

Table 1: Performance metrics for the GP-based variants. For each covariance function and each QoI: the empirical coverage is given first, and then (in blue) the ratio $\Delta / \Delta_{\text {GCI }}$ of the IHW of the GP-based method by the IHW of the GCI-based method. $\mathcal{M}_{\nu}$ denotes the Matérn covariance with regularity $\nu$. The sad face $;$ indicates that either the coverage is below $80 \%$ or $\Delta>3 \Delta_{\text {GCI }}$.

\section{CONCLUSIONS}

The Bayesian (GP-based) approach to the quantification of discretization uncertainty emerges from this study as a promising alternative to the GCI approach, with the potential to provide "well-founded probability statements" and, ultimately, better confidence intervals (i.e., shorter intervals that still have a satisfactory coverage).

At the present time, however, the Bayesian approach lacks the maturity and robustness of the GCI approach: which covariance model to use, and how to robustly select the hyper-parameters (e.g, the decay parameter $L$ in the TWY2 model), are important questions that deserve further attention. In a different direction, the construction of (possibly sequential) DoEs, both for gridrefinement studies as considered in this article, and for the more general case of parametric studies, is also an interesting direction for future work.

\section{References}

[1] R. J. Adler. The Geometry of Random Fields. Wiley, New York, 1981.

[2] M. S. Alnæs, J. Blechta, J. Hake, A. Johansson, B. Kehlet, A. Logg, C. Richardson, J. Ring, M. E. Rognes, and G. N. Wells. The fenics project version 1.5. Archive of Numerical Software, 3(100), 2015.

[3] C. E. Augarde and A. J. Deeks. The use of timoshenko's exact solution for a cantilever beam in adaptive analysis. Finite elements in analysis and design, 44(9-10):595-601, 2008.

[4] J. Bect, E. Vazquez, et al. STK: a Small (Matlab/Octave) Toolbox for Kriging. Release 2.6.1, 2019. URL http://kriging. sourceforge.net.

[5] C. Currin, T. J. Mitchell, M. Morris, and D. Ylvisaker. Bayesian prediction of deterministic functions, with applications to the design and analysis of computer experiments. Journal of the American Statistical Association, 86(416):953-963, 1991.

[6] M.C. Kennedy and A. O'Hagan. Bayesian calibration of computer models. Journal of the Royal Statistical Society. Series B Statistical Methodology, 63(3):425-464, 2001. 
[7] W. L. Oberkampf and C. J. Roy. Verification and validation in scientific computing. Cambridge University Press, 2010.

[8] V. Picheny and D. Ginsbourger. A nonstationary space-time Gaussian process model for partially converged simulations. SIAM/ASA Journal on Uncertainty Quantification, 1(1): 57-78, 2013.

[9] L. F. Richardson. The approximate arithmetical solution by finite differences of physical problems involving differential equations, with an application to the stresses in a masonry dam. Philosophical Transactions of the Royal Society of London. Series A, Containing Papers of a Mathematical or Physical Character, 210(459-470):307-357, 1911.

[10] L. F. Richardson and J. A. Gaunt. The deferred approach to the limit. Philosophical Transactions of the Royal Society of London. Series A, containing papers of a mathematical or physical character, 226(636-646):299-361, 1927.

[11] P. J. Roache. Perspective: a method for uniform reporting of grid refinement studies. Journal of Fluids Engineering, 116(3):405-413, 1994.

[12] P. J. Roache. Quantification of uncertainty in computational fluid dynamics. Annual Review of Fluid Mechanics, 29(1):123-160, 1997.

[13] P. J. Roache. Verification of codes and calculations. AIAA Journal, 36(5):696-702, 1998.

[14] J. Sacks, S. B. Schiller, and W. J. Welch. Designs for computer experiments. Technometrics, 31(1):41-47, 1989.

[15] J. Sacks, W. J. Welch, Mitchell T. J., and H. P. Wynn. Design and analysis of computer experiments. Statistical Science, 4(4):409-435, 1989.

[16] M. L. Stein. Interpolation of Spatial Data: Some Theory for Kriging. Springer Series in Statistics. Springer, New York, 1999.

[17] R. Stroh, J. Bect, S. Demeyer, N. Fischer, and E. Vazquez. Integrating hyper-parameter uncertainties in a multi-fidelity Bayesian model for the estimation of a probability of failure, pages 349-356. World Scientific.

[18] R. Stroh, J. Bect, S. Demeyer, N. Fischer, D. Marquis, and E. Vazquez. Assessing fire safety using complex numerical models with a Bayesian multi-fidelity approach. Fire Safety Journal, 91:1016-1025, 2017.

[19] R. Tuo, C. F. Jeff Wu, and D. Yu. Surrogate modeling of computer experiments with different mesh densities. Technometrics, 56(3):372-380, 2014.

[20] W. Weaver, S. P. Timoshenko, and D. H. Young. Vibration Problems in Engineering. John Wiley \& Sons, 1990.

[21] W. J. Welch, R. J. Buck, J. Sacks, H. P. Wynn, T. J. Mitchell, and M. D. Morris. Screening, predicting and computer experiments. Technometrics, 34:15-25, 1992. 